Pacific Journal of Mathematics

COLLECTIONS OF COVERS OF METRIC SPACES 


\title{
COLLECTIONS OF COVERS OF METRIC SPACES
}

\author{
TED R. PetTis
}

\begin{abstract}
In this paper cardinality $\kappa$ collections of open covers of a topological space satisfying various conditions are studied. When $\kappa=\omega$ some of the conditions are equivalent to the space being metrizable and the union of a compact set and a discrete set. For a metrizable space some of the conditions are equivalent to complete metrizability. If $\kappa \neq \omega$ then the relationship between some of the conditions and the existence of scales is examined.
\end{abstract}

\section{Introduction and definitions.}

1.1. An ordinal number is the set of all ordinals which precede it and a cardinal number is an ordinal which cannot be put in a one-to-one correspondence with any ordinal which precedes it. Throughout this paper $\omega$ will denote the set of all finite ordinals and $\kappa$ will denote an infinite cardinal number.

If $M$ is a set, $x$ is a point, and $\mathscr{C}$ is a collection of sets, then the star of $M$. with respect to $\mathscr{K}$, denoted $s t(M, \mathscr{C})$ is the union of all members of $\mathscr{K}$ which meet $M$ and $s t(x, \mathscr{H})=s t(\{x\}, \mathscr{H})$. A sequence $\mathscr{G}=\mathscr{G}_{0}, \mathscr{G}_{1}, \mathscr{G}_{2}, \cdots$ of open covers of a topological space $S$ is called a development for $S$ iff for each $x \in S$ and open set $U$ containing $x$ there is an $n$ such that $s t\left(x, \mathscr{G}_{n}\right) \subseteq U$. Moreover, a development is monotonic iff $\mathscr{G}_{n+1} \subseteq \mathscr{G}_{n}$ for all $n$. A space which admits a development is called a developable space and a regular- $T_{1}$ developable space is called a Moore space. A development $\mathscr{G}$ for a Moore space is star complete (see [16]) provided that if $\left\{M_{0}, M_{1}\right.$, $\left.M_{2}, \cdots\right\}$ is a sequence of closed sets such that for each $n, M_{n+1} \subseteq$ $M_{n} \leqq s t\left(x, \mathscr{G}_{n}\right)$ for some $x \in S$ then $\cap M_{n} \neq \varnothing$. A Moore space having a star complete development is said to be star complete. A Moore space $S$ is Moore-closed (see [5] and [6]) iff $S$ is closed in each Moore space in which $S$ is embedded.

A space $S$ is a $w \Delta$-space (see [3]) iff there exists a sequence $\mathscr{B}_{0}, \mathscr{\mathscr { B }}_{1}, \mathscr{B}_{2}, \cdots$ of open covers of $S$ such that for each $x \in S$, if $x_{n} \in$ $s t\left(x, \mathscr{B}_{n}\right)$ then the sequence $\left\{x_{0}, x_{1}, x_{2}, \cdots\right\}$ has a cluster point. A space $S$ has a $G_{o i}^{*}$-diagonal (see [10]) provided there is a sequence $\mathscr{G}_{0}, \mathscr{G}_{1}$, $\mathscr{B}_{2}, \cdots$ of open covers of $S$ such that if $x$ and $y$ are distinct points of $S$, there is an $n$ such that $y \notin s \overline{s\left(x, \mathscr{G}_{n}\right)}$.

A nonempty subset $M$ of a topological space $S$ is called discrete iff for each $x \in M$ there is an open set $U$ such that $U \cap M=\{x\} . \quad$ A collection of sets is discrete if the closures of the sets are mutually 
exclusive and the union of any subcollection of these closures is closed. A topological space is $\kappa$-collectionwise Hausdorff iff for every closed discrete subset $M$ having cardinality at most $\kappa$, there is a pairwise disjoint collection of open sets covering $M$, no member of which contains more than one point of $M$. A space which is $\kappa$-collectionwise Hausdorff for all cardinals $\kappa$ is called collectionwise Hausdorff. Regular- $T_{1}$ spaces are $\omega$-collectionwise Hausdorff.

1.2. For a topological space $S, \mathscr{D}(S)$ denotes the collection of all closed discrete subsets of $S$ and $\mathscr{D}^{*}(S)$ denotes the collection of all infinite closed, discrete subsets of $S$. Consider the following conditions on a cardinality $\kappa$ collection $\mathscr{G}=\left\{\mathscr{G}_{\alpha}: \alpha \in \kappa\right\}$ of open covers of $S$.

Condition $\mathrm{A}(\kappa)$. For each $D \in \mathscr{D}(S)$ and open set $U$ containing $D$ there exists an $\alpha \in \kappa$ such that $\operatorname{st}\left(D, \mathscr{G}_{\alpha}\right) \cong U$.

Condition $\mathrm{wA}(\kappa)$. For each $D \in \mathscr{D}^{*}(S)$ and open set $U$ containing $D$ there exists an infinite subset $D^{\prime}$ of $D$ and an $\alpha \in \kappa$ such that $\operatorname{st}\left(D^{\prime}, \mathscr{G}_{\alpha}\right) \subseteq U$.

Condition $\mathrm{B}(\kappa)$. For each $D \in \mathscr{D}(S)$ there is an $\alpha \in \kappa$ such that if $x$ and $y$ are distinct points of $D$, then $s t\left(x, \mathscr{G}_{\alpha}\right) \cap s t\left(y, \mathscr{G}_{\alpha}\right)=\varnothing$.

Condition $\mathrm{wB}(\kappa)$. For each $D \in \mathscr{D}^{*}(S)$ there exists an infinite subset $D^{\prime}$ of $D$ and an $\alpha \in \kappa$ such that if $x$ and $y$ are distinct points of $D^{\prime}$ then $s t\left(x, \mathscr{G}_{\alpha}\right) \cap s t\left(y, \mathscr{G}_{\alpha}\right)=\varnothing$.

Condition $\mathrm{C}(\kappa)$. For each $D \in \mathscr{D}(S)$ and $E \in \mathscr{D}(S)$ with $D \cap E=$ $\varnothing$ there exists an $\alpha \in \kappa$ such that $\operatorname{st}\left(D, \mathscr{G}_{\alpha}\right) \cap \operatorname{st}\left(E, \mathscr{G}_{\alpha}\right)=\varnothing$.

Condition $\mathrm{wC}(\kappa)$. For each $D \in \mathscr{D}^{*}(S)$ and $E \in \mathscr{D}(S)$ with $D \cap$ $E=\varnothing$ there exists an infinite subset $D^{\prime}$ of $D$ and an $\alpha \in \kappa$ such that $s t\left(D^{\prime}, \mathscr{G}_{\alpha}\right) \cap s t\left(E, \mathscr{G}_{\alpha}\right)=\varnothing$.

Condition $\mathrm{wwC}^{(\kappa)}$. For each $D \in \mathscr{D}^{*}(S)$ and $E \in \mathscr{D}^{*}(S)$ with $D \cap E=\varnothing$ there exist infinite subsets $D^{\prime}$ and $E^{\prime}$ of $D$ and $E$ respectively and an $\alpha \in \kappa$ such that $\operatorname{st}\left(D^{\prime}, \mathscr{G}_{\alpha}\right) \cap \operatorname{st}\left(E^{\prime}, \mathscr{G}_{\alpha}\right)=\varnothing$.

A space is said to satisfy one of the conditions above if it admits a collection of open covers which satisfies the condition. For the case where $\kappa=\omega$ reference to $\omega$ is dropped whence by condition A is meant condition $\mathrm{A}(\omega)$ and so forth.

\section{Moore spaces.}

2.1. If $S$ is a developable space which has a collection of open covers satisfying a condition defined in 1.2 for $\kappa=\omega$ then $S$ has a monotonic development satisfying that condition.

THEOREM 2.2. For a regular $T_{1}$-space $S$ containing no infinite open and closed discrete subset the following statements are equivalent. 
(1) $S$ is a compact metric space.

(2) $S$ is a Moore space and every infinite subset of $S$ has a limit point.

(3) S satisfies condition A.

(4) $S$ satisfies condition B.

(5) S satisfies condition C.

(6) $S$ is developable and satisfies condition wA.

(7) $S$ is developable and satisfies condition $\mathrm{wC}$.

(8) $S$ is Moore-closed and satisfies condition wB.

(9) $S$ is Moore-closed and satisfies condition wwC.

Proof. That (1) is equivalent of (2) is well known and that (1) implies each of the conditions (3) through (9) is immediate.

$(3) \rightarrow(2)$. A sequence of covers satisfying condition $\mathrm{A}$ is a development; thus $S$ is a Moore space. Let $\mathscr{G}$ be a monotonic development for $S$ satisfying condition A. Suppose $M=\left\{x_{0}, x_{1}, x_{2}, \cdots\right\}$ is an infinite set of limit points of $S$ and $M$ fails to have a limit point. Since $S$ is $\omega$-collectionwise Hausdorff there is a pairwise disjoint collection of open sets $U_{0}, U_{1}, U_{2}, \cdots$ with $x_{0} \in U_{0}, x_{1} \in U_{1}, \cdots$. For each $n$, let $y_{n} \in \operatorname{st}\left(x_{n}, \mathscr{V}_{n}\right) \cap U_{n}-\left\{x_{n}\right\}$. Then $S-\operatorname{cl}\left\{y_{0}, y_{1}, y_{2}, \cdots\right\}$ is an open set containing $M$ but there does not exist an $n$ such that $\operatorname{st}\left(M, \mathscr{O}_{n}\right) \subseteq S-\operatorname{cl}\left\{y_{0}, y_{1}, y_{2}, \cdots\right\}$. Thus $M$ must have a limit point.

$(4) \rightarrow(2)$. It follows immediately from the definitions that a space satisfying condition $\mathrm{B}$ is a $w \Delta$ space with a $G_{\dot{0}}^{*}$-diagonal and hence is developable. Let be a monotonic development satisfying condition $\mathrm{B}$ and let $M$ and $\left\{y_{0}, y_{1}, y_{2}, \cdots\right\}$ be as in the proof of $(3) \rightarrow$ (2). Then either $\left\{y_{0}, y_{1}, y_{2}, \cdots\right\}$ is closed in which case $M^{\prime}=M \cup$ $\left\{y_{0}, y_{1}, \cdots\right\}$ is a set for which condition B fails or $\left\{y_{0}, y_{1}, \cdots\right\}$ has a limit point $y$ in which case $M^{\prime}=M \cup\{y\}$ is a set for which condition $B$ fails.

$(5) \rightarrow(2)$. The proof is similar to $(4) \rightarrow(2)$.

$(6) \rightarrow(2)$. The same proof as $(3) \rightarrow(2)$.

$(7) \rightarrow(2)$. Similar to $(4) \rightarrow(2)$.

$(8) \rightarrow(1)$. If $\mathscr{C}$ is a monotonic development for $S$ satisfying condition wB then it is a star complete development. For if not, there exists a sequence $\left\{M_{0}, M_{1}, M_{2}, \cdots\right\}$ of closed sets such that $M_{n+1} \subseteq M_{n}$ and a sequence $\left\{x_{0}, x_{1}, x_{2}, \cdots\right\}$ of points of $S$ such that $M_{n} \sqsubseteq s t\left(x_{n}, \mathscr{G}_{n}\right)$ for all $n$ and $\cap M_{n}=\varnothing$. Without loss of generality it may be assumed that $M_{n}-M_{n+1} \neq \varnothing$ for all $n$. Let $y_{n} \in M_{n}-M_{n+1}$. Then $\left\{y_{0}, y_{1}, y_{2}, \cdots\right\} \in \mathscr{D}^{*}(S)$. Condition $\mathrm{wB}$ fails to hold for this set. A star complete Moore-closed space is compact ([6, Theorem 1.5]).

$(9) \rightarrow(1)$. Similar to $(8) \rightarrow(1)$. 
2.3. Since a Moore space which is the union of a compact set and a discrete set is paracompact the next theorem follows immediately from the proof of Theorem 2.2. See Theorem 1.6 of [6] for a related result.

THEOREM 2.4. For a regular $T_{1}$-space $S$ the following statements are equivalent.

(1) $S$ is a Moore space which is the union of a compact set and a discrete set.

(2) $S$ is a metric space and the set of all limit points of $S$ is compact.

(3) S satisfies condition A.

(4) S satisfies condition B.

(5) $S$ satisfies condition C.

(6) $S$ is developable and satisfies condition wA.

(7) $S$ is developable and satisfies condition wC.

2.3. The next theorem shows that Moore-closed was needed as part of the hypothesis in Theorem 2.2.

THEOREM 2.5. For a metrizable space $S$ the following statements are equivalent.

(1) S has a complete metric.

(2) $S$ satisfies condition $\mathrm{wB}$.

(3) S satisfies condition wwC.

Proof. By the proof of Theorem 2.2 if $S$ satisfies condition wB or condition wwC then $S$ is star complete and hence complete Moore and thus has a complete metric by the result of Roberts [15].

$(1) \rightarrow(2)$. Suppose $S$ has a complete metric $d$. Let $B(x, \varepsilon)=$ $\{y \in S: d(x, y)<\varepsilon\}$.

For each $n$ let $\mathscr{G}_{n}=\left\{B\left(x, 1 / 2^{n+1}\right): n \in \omega\right\}$. Let $M$ be a countably infinite, closed, discrete subset of $S$. For each

$$
n, A_{n}=\left\{x \in M: \operatorname{st}\left(s t\left(x, \mathscr{G}_{n}\right), \mathscr{G}_{n}\right) \cap M \text { is finite }\right\} .
$$

Suppose $A_{n}$ is finite for each $n$. There is a point $x_{0} \in M-A_{0}$. Then let $x_{n+1} \in \operatorname{st}\left(s t\left(x_{n}, \mathscr{G}_{n}\right), \mathscr{G}_{n}\right) \cap M-A_{n+1}-\left\{x_{0}, x_{1}, \cdots, x_{n}\right\}$. The sequence $\left\{x_{0}, x_{1}, x_{2}, \cdots\right\}$ is Cauchy and hence converges to a point $y$. Thus $y$ is a limit point of $M$ which is impossible. Thus, there is a $k$ such that $A_{k}$ is infinite. Let $\left\{a_{0}, a_{1}, a_{2}, \cdots\right\}$ be the points of $A_{k}$. There is a least positive integer $n_{1}$ such that $a_{n_{1}} \notin \operatorname{st}\left(\operatorname{st}\left(a_{0}, \mathscr{O}_{k}\right), \mathscr{G}_{k}\right)$. There is a least positive integer $n_{2}$ such that $a_{n_{2}} \notin \operatorname{st}\left(s t\left(a_{0}, a_{n_{1}}, \mathscr{G}_{k}\right), \mathscr{G}_{k}\right)$. This process may be continued. The set $A=\left\{a_{0}, a_{n_{1}}, a_{n_{2}}, \cdots\right\}$ has the required property. 
The proof of $(1) \rightarrow(3)$ is similar.

\section{First countable spaces.}

3.1. An interesting generalization of development due to E. E. Grace [1], is the concept of a quasi-development. A sequence $\mathscr{G}=$ $\mathscr{O}_{0}, \mathscr{G}_{1}, \mathscr{G}_{2}, \cdots$ of collections of open subsets of a topological space $S$ is called a quasi-development for $S$ provided for each point $p$ of $S$ and open set $U$ containing $p$ there is an $n$ such that $\operatorname{st}\left(p, \mathscr{G}_{n}\right) \neq \varnothing$ and $s t\left(p, \mathscr{G}_{n}\right) \subseteq U$. The conditions defined in 1.2 can be modified to conditions on quasi-developments by requiring that the collection $\mathscr{G}_{n}$ in the definition cover the closed discrete sets. The following theorem results from these modifications.

THeOREm 3.2. For a regular $T_{1}$ space $S$ containing no infinite, discrete, closed and open subset, the following statements are equivalent.

(1) $S$ is compact metric.

(2) S has a quasi-development satisfying condition A.

(3) S has a quasi-development satisfying condition B.

(4) S has a quasi-development satisfying condition C.

(5) S has a quasi-development satisfying condition wA.

(6) $S$ has a quasi-development satisfying condition $\mathrm{wC}$.

Proof. That (1) implies each of statements (2) through (6) is immediate.

By modifying the arguments of Theorem 2.2 only slightly, it can be shown that each of statements (2) through (6) implies that $S$ is countably compact. By a result of Wicke and Worrell [17, Theorem 2.10] countably compact quasi-developable spaces are compact.

3.3. Another generalization of the results of $\S 2$ is obtained in the following fashion. Using the notation of Hodel in [11] let $(S, \tau)$ be a regular $T_{1}$ space, and let $g: S \times \omega \rightarrow \tau$ be a function such that $x \in g(x, n)$ for all $x \in S$ and $n \in \omega$ and such that $g(x, n+1) \leqq g(x, n)$ for all $n$. If $D$ is a nonempty set then $g^{*}(D, n)=\cup\{g(x, n): x \in D\}$. If $C$ is one of the conditions defined in $\S 1.2$ and $\operatorname{st}\left(x, \mathscr{G}_{n}\right)$ and $\operatorname{st}\left(D, \mathscr{G}_{n}\right)$ are replaced by $g(x, n)$ and $g^{*}(D, n)$, respectively, the resulting statement defines $g$ to have condition C. The next theorems follow from the corresponding proofs in $\S 2$.

THeOREM 3.4. For a regular $T_{1}$ space $S$ containing no infinite, open and closed subset, the following statements are equivalent.

(1) $S$ is a first countable, countably compact space. 
(2) There is a function $g$ for $S$ which satisfies condition A.

(3) There is a function $g$ for $S$ which satisfies condition B.

(4) There is a function $g$ for $S$ which satisfies condition C.

(5) $S$ is first countable and there is a function $g$ for $S$ which satisfies condition wA.

(6) $S$ is first countable and there is a function $g$ for $S$ which satisfies condition $\mathrm{wC}$.

TheOREM 3.5. For a first countable, regular $T_{1}$ space $S$ the following statements are equivalent:

(1) The set of all limit points of $S$ is countably compact.

(2) Then is a function $g$ for $S$ which satisfies condition A.

(3) There is a function $g$ for $S$ which satisfies condition B.

(4) There is a function $g$ for $S$ which satisfies condition C.

(5) There is a function $g$ for $S$ which satisfies condition wA.

(6) There is a function $g$ for $S$ which satisfies condition $\mathrm{wC}$.

\section{Uncountable collections.}

4.1. If each definition in $\S 1.2$ is viewed as a cardinal function, a natural question is what is the minimum cardinal $\kappa$ such that a space $S$ admits a cardinality $\kappa$ collection of open covers satisfying that condition? Also one might ask if a space $S$ admits a cardinality $\kappa$ collection of open covers satisfying a condition in 1.2 what does this imply about $S$ ? In this section some partial answers to these two questions are given.

A topological space $S$ is said to have property $D(\kappa)$ iff for each closed discrete subset $M$ of $S$ with cardinality at most $\kappa$ there is a collection $\mathscr{H}$ of mutually exclusive open sets such that (1) $\mathscr{C}$ covers $M$ and each member of $\mathscr{C}$ contains only one point of $M$, and (2) if $N$ is a set covered by $\mathscr{C}$ such that each member of $\mathscr{C}$ contains only one point of $N$ then $N$ has no limit point. A space which has property $D(\omega)$ is said to have property $D$ (see [13, page 69]). For an infinite cardinal $\kappa$ a space having the property that each of its subsets of cardinality $\kappa$ has a limit point will be called $\kappa$-compact.

THEOREM 4.2. If $\kappa$ is an infinite cardinal, and $S$ is a regular. $T_{1}$ space which has property $D(\kappa)$, which satisfies at least one of the conditions $A(\kappa), B(\kappa)$, or $C(\kappa)$, and which contains no infinite discrete, open and closed subset of cardinality $\kappa$, then $S$ is $\kappa$-compact.

Proof. Suppose the theorem is false. Then there exists a closed, discrete subset $M$ of limit points of $S$. Let $\mathscr{C}$ be an open cover of $S$ satisfying (1) and (2) of the definition of property $D(\kappa)$. Pro- 
ceeding as in the proof of Theorem 3.2 will yield a contradiction.

4.3. Notation and definitions from set theory not stated here may be found in [12]. The usual axioms for set theory, the ZermeloFraenkel axioms including the axiom of choice, will be denoted by $\mathrm{ZFC}$, the continuum hypothesis will be denoted by $\mathrm{CH}$, and Martin's axiom will be denoted by MA. If $\alpha$ is a limit ordinal, the cofinality of $\alpha$ denoted $\operatorname{cf}(\alpha)$, is the least ordinal $\beta$ such that there is a function $f$ from $\beta$ into $\alpha$ such that $\sup \{f(x): x \in \beta\}=\alpha$.

The set " $\omega$ of all functions from $\omega$ to $\omega$ has two natural orders. If $f$ and $g$ are functions from $\omega$ to $\omega$ then $f<g$ iff $f(n)<g(n)$ for each $n \in \omega$, and $f<{ }^{*} g$ iff there is an $m$ such that $f(n)<g(n)$ for all $n>m$. A subset $\mathscr{S}$ of " $\omega$ is called a scale provided for each $f \in{ }^{\prime \prime} \omega$ there is a $g \in \mathscr{S}$ such that $f<* g$. A subset $\mathscr{S}$ of " $\omega$ is called a dominating family iff for each $f \in{ }^{\omega} \omega$ there is a $g \in \mathscr{S}$ such that $f<g$. If there is a scale with cardinality $\kappa$ there is a dominating family of cardinality $\kappa$. See [8] for results on the existence of scales. Among the results there, Hechler shows that MA implies all scales have cardinality $c$ and for each cardinal $\kappa$ such that $\omega_{1} \leqq$ $\operatorname{cf}(\kappa) \leqq \kappa \leqq c$, it is consistent with $\mathrm{ZFC}$ that there exists a scale whose cardinality is $\kappa$.

REMARK 4.4. Whether the converse to Theorem 4.2 is true, even for metric spaces, depends on the type of set theory assumed. The case for $\omega_{1}$-compactness is of particular interest, since in metric spaces $\omega_{1}$-compact, Lindelof, separable, and second countable are equivalent and important.

THEOREM 4.5. $\mathrm{CH}$ implies that if $S$ is a metric space which has no uncountable, discrete, closed and open subset, the following are equivalent.

(1) $S$ is $\omega_{1}$-compact.

(2) S satisfies condition $\mathrm{A}\left(\omega_{1}\right)$.

(3) $S$ satisfies condition $\mathrm{B}\left(\omega_{1}\right)$.

(4) $S$ satisfies condition $\mathrm{C}\left(\omega_{1}\right)$.

Proof. An argument similar to the one used in the proof of Theorem 2.2 will establish each of the implications $(2) \rightarrow(1),(3) \rightarrow$ (1) and (4) $\rightarrow(1)$. That (1) implies each of the statements (2), (3), and (4) follows from the fact that an $\omega_{1}$-compact metric space is second countable.

EXAMPLE 4.6. If $\kappa<c f(c)$, there is a subspace $S$ of the real line such that if $=\left\{\mathscr{S}_{n}: \alpha \in \kappa\right\}$ is a collection of open covers of $S$, 
there is a $D \in \mathscr{D}(S)$ such that if $\beta \in \kappa$, there is a member of $\mathscr{C}_{\beta}$ containing more than one point of $D$. Thus $S$ does not satisfy condition $\mathrm{A}(\kappa)$ or condition $\mathrm{B}(\kappa)$. A modification of the argument which follows shows that $S$ does not satisfy condition $\mathrm{C}(\kappa)$ either. There is a subset $S$ of the set $R$ of real numbers such that both $S$ and $R-S$ have cardinality $c$ and, moreover, both $S$ and $R-S$ intersect every uncountable, closed subset of $R$ [4]. Suppose there is a collection $\mathscr{G}=\left\{\mathscr{G}_{\alpha}: \alpha \in \kappa\right\}$ of open covers of $S$ contrary to the claim. For each point $t \in R-S$ there is a sequence $\left\{t_{0}, t_{1}, t_{2}, \cdots\right\}$ of points of $S$ which converges to $t$. The set of terms of this sequence is discrete and closed in the subspace topology on $S$. For each $\alpha \in \kappa$, let $T_{\alpha}$ be the set of all points $t$ belonging to $R-S$ such that no member of $\mathscr{G}_{\alpha}$ contains more that one point of the sequence $\left\{t_{0}, t_{1}\right.$, $\left.t_{2}, \cdots\right\}$. Since $c f(c)>\kappa$ and $\bigcup_{\alpha \in \kappa} T_{\alpha}=R-S$, for some $\alpha \in \kappa, T_{\alpha}$ has cardinality $c$. The closure in $R$ of $T_{\alpha}$ contains a point $p$ of $S$. The point $p$ belongs to some member $V$ of $\mathscr{G}_{\alpha}$. There is a set $U$ open in $R$ such that $V=U \cap S$. Moreover, $U \cap T_{\alpha} \neq \varnothing$. If $t \in U \cap T_{\alpha}, U$ contains a tail of the sequence $\left\{t_{0}, t_{1}, t_{2}, \cdots\right\}$ associated with $t$, but then so does $V$ and this is a contradiction.

4.7. In what follows the space $Y$ will denote the set to which a point $x$ belongs iff $x$ is a nonnegative integer or for nonnegative integers $n$ and $k, x=n-1 /(k+2)$. The topology on $Y$ is the subspace topology $Y$ inherits as a closed subset of the set of real numbers with the usual topology.

Lemma 4.8. If $S$ is a metric space and the set of all limit points of $S$ is not compact, then $S$ includes a closed subspace which is homeomorphic to the space $Y$.

LEMma 4.9. If $\kappa$ is an infinite cardinal; there is a cardinality $\kappa$ collection of open cover's of $Y$ which satisfies at least one of conditions $\mathrm{A}(\kappa), \mathrm{B}(\kappa)$ or $\mathrm{C}(\kappa)$ iff there is a dominating family of cardinality $\kappa$.

Proof. If $\mathscr{C}=\left\{\mathscr{C}_{\alpha}: \alpha \in \kappa\right\}$ is a collection of open covers of $Y$, define $f_{\alpha}$ as follows. For each $n$, let $f_{\alpha}(n)=\inf \{i: n-1 /(i+2) \epsilon$ $\left.\operatorname{st}\left(n, \mathscr{C}_{\alpha}\right)\right\}$. The set $\left\{f_{\alpha}: \alpha \in \kappa\right\}$ forms a dominating family provided the collection of covers $\left\{\mathscr{G}_{\alpha}: \alpha \in \kappa\right\}$ satisfies condition $\mathrm{A}(\kappa)$ or $\mathrm{B}(\kappa)$ or $\mathrm{C}(\kappa)$.

Conversely, if $n$ and $k$ are nonnegative integers and $f \in{ }^{\omega} \omega$, let $U(n, k)=\{n\} \cup\{n-1 /(i+2): i \geqq k\}$ and $\mathscr{G}_{f}=\{\{n-1 /(k+2)\}: n, k \in \omega\} \cup$ $\{U(n, f(n)): n \in \omega\}$. If $\mathscr{S}$ is a dominating family of cardinality $\kappa$, then $\left\{G_{f}: f \in \mathscr{S}\right\}$ is a cardinality $\kappa$ collection of open covers satisfying conditions $\mathrm{A}(\kappa), \mathrm{B}(\kappa)$ and $\mathrm{C}(\kappa)$. 
THEOREM 4.10. If $\kappa$ is an infinite cardinal and $S$ is a $\sigma$-compact metric space whose set of limit points is not compact, the following statements are equivalent.

(1) There is a dominating family of cardinality $\kappa$.

(2) $S$ satisfies condition $\mathrm{A}(\kappa)$.

(3) $S$ satisfies condition $\mathrm{B}(\kappa)$.

(4) $S$ satisfies condition $\mathrm{C}(\kappa)$.

Proof. Suppose there is a dominating family $\mathscr{S}$ having cardinality $\kappa$. There exists an increasing sequence $\left\{F_{0}, F_{1}, F_{2}, \cdots\right\}$ of compact sets whose union is $S$. For each pair of nonnegative integers $n$ and $k$, let $\mathscr{S}_{n}^{k}$ be a cover of $F_{n}$ by open balls, centered at a point of $F_{n}$, with radius less than $1 / 2^{k+1}$. For each $g \in \mathscr{S}$ define $\mathscr{O}_{g}=\left\{\mathscr{G}_{n}^{g(n)}: n \in \omega\right\}$. If $D \in \mathscr{D}(S)$ there is a function $f \in{ }^{\omega} \omega$ such that if $x$ and $y$ are distinct points of $D$, at least one of which belongs to $D$, then $d(x, y)>1 / 2^{f(n)}$. It is then easily seen that $\mathscr{G}=\left\{\mathscr{G}_{g}: g \in \mathscr{S}\right\}$ satisfies each of conditions $\mathrm{A}(\kappa), \mathrm{B}(\kappa)$, and $\mathrm{C}(\kappa)$.

To prove the implications $(4) \rightarrow(1),(3) \rightarrow(1),(2) \rightarrow(1)$, note that $S$ includes a closed subspace homeomorphic to $Y$. Each of conditions $\mathrm{A}(\kappa), B(\kappa)$ and $\mathrm{C}(\kappa)$ is hereditary on closed subsets. Lemma 4.9 gives the desired result.

REMARK 4.11. Example 4.6 shows that Theorem 4.10 does not hold in general for all metric spaces. The next result improves 4.10 slightly, but the collections of sets are no longer covers.

THEOREM 4.12. If there is a dominating family with cardinality $\omega_{1}$ and $S$ is an $\omega_{1}$-compact metric space which is the union of $\omega_{1}$, compact sets, there is a collection $\mathscr{G}$ of each type below.

(1) $\mathscr{G}=\left\{\mathscr{G}_{\alpha}: \alpha \in \omega_{1}\right\}$ is a collection of sets of open subsets of $S$ having the property that if $D \in \mathscr{D}(S)$ and $U$ is an open set including $D$, there is an $\alpha \in \omega_{1}$ such that $\mathscr{G}_{\alpha}$ covers $D$ and $\operatorname{st}\left(D, \mathscr{G}_{\alpha}\right) \subseteq U$.

(2) $\mathscr{G}=\left\{\mathscr{G}_{\alpha}: \alpha \in \omega_{1}\right\}$ is a collection of sets of open subsets of $S$ having the property that if $D \in \mathscr{D}(S)$, there is an $\alpha \in \omega_{1}$ such that $\mathscr{G}_{\alpha}$ covers $D$ and if $x$ and $y$ are distinct points of $D$, then $\operatorname{st}\left(x, \mathscr{G}_{\alpha}\right) \cap s t\left(y, \mathscr{G}_{\alpha}\right)=\varnothing$.

(3) $\mathscr{S}=\left\{\mathscr{C}_{\alpha}: \alpha \in \omega_{1}\right\}$ is a collection of sets of open subsets of $S$ having the property that if $D$ and $E$ are pairwise disjoint sets, $D \in \mathscr{D}(S)$, and $E \in \mathscr{D}(S)$ there is an $\alpha \in \omega_{1}$ such that $\mathscr{G}_{\alpha}$ covers $D \cup E$ and $\operatorname{st}\left(D, \mathscr{C}_{\alpha}\right) \cap \operatorname{st}\left(E, \mathscr{O}_{\alpha}\right)=\varnothing$.

Proof. There is a collection $\left\{F_{\alpha}: \alpha \in \omega_{1}\right\}$ of compact subsets of $S$ whose union is $S$. For each $\alpha \in \omega_{1}$ the collection $\left\{F_{\beta}: \beta \in \alpha\right\}$ is a countable collection of compact sets and if $D \in \mathscr{D}(S)$ there is an 
$\alpha \in \omega_{1}$ such that $\left\{F_{\beta}: \beta \in \alpha\right\}$ covers $D$. The construction in 4.10 applied to this collection yields a cardinality $\omega_{1}$ collection of open covers of $\cup\left\{F_{\beta}: \beta \in \alpha\right\}$. Then the family of all collections for all $\beta \in \omega_{1}$ satisfies each of conditions (1), (2), and (3) and has cardinality $\omega_{1}$.

REMARK 4.13. If there is a dominating family of cardinality $\omega_{1}$, then the irrationals-indeed, every metric space which is the continuous image of the irrationals-is $\omega_{1}$-compact and is the union of a cardinality $\omega_{1}$ collection of compact sets (see [9]). If $\mathrm{CH}$ is false then the space of Example 4.6 is not the union of a cardinality $\omega_{1}$ collection of compact sets.

\section{An application for the set of real numbers.}

5.1. A scale $\mathscr{S}$ which is order isomorphic to the ordinal $\alpha$ is called an $\alpha$-scale. Hausdorff in [7] showed that $\mathrm{CH}$ implies there is an $\omega_{1}$-scale. The set of rational real numbers is denoted $Q$, the set of irrational real numbers is denoted $P$, and the set of real numbers is denoted $R$. $\mathscr{F}$ will denote the set $\mathscr{D}(Q) \cap \mathscr{D}(R)$.

THEOREM 5.2. If there is a dominating family of cardinality $\omega_{1}$, and $\omega_{1}<c f(c)$, then every subcollection $\mathscr{P}$ of $\mathscr{F}$ with cardinality $c$ has a subcollection $\mathscr{C}^{\prime}$ with cardinality $c$, and such that $\cup \mathscr{K}^{\prime}$ has no irrational limit point.

Proof. It follows with the aid of Theorem 4.10 there is a collection $\mathscr{G}=\left\{\mathscr{G}_{\alpha}: \alpha \in \omega_{1}\right\}$ of open covers of $R$ satisfying condition $B\left(\omega_{1}\right)$ and such that for each $\alpha, G_{\alpha}$ is countable and locally finite. For $\alpha \in \omega_{1}$ let $\mathscr{G}_{\alpha}=\left\{V_{n}: n \in \omega\right\}$. For each $n, x_{0}^{n}, x_{1}^{n}, x_{2}^{n}, \cdots$ are the points of the set $Q \cap V_{n}$. There is a dominating family $\mathscr{S}$ with cardinality $\omega_{1}$. For $f \in \mathscr{S}$ define $D_{f}^{\alpha}=\bigcup_{n \in \omega}\left\{x_{t}^{n}: t \leqq f(n)\right\}$. The set $\mathscr{C}=\left\{D_{f}^{\alpha}: \alpha \in \omega_{1}\right.$ and $\left.f \in \mathscr{S}\right\}$ has cardinality at most $\omega_{1}$. Each $D_{f}^{\alpha}$ is a closed discrete subset of $R$. Moreover, $\mathscr{C}$ has the property that if $F \in \mathscr{F}$ there is an $\alpha \in \omega_{1}$ and an $f \in \mathscr{S}$ such that $F \subseteq D_{f}^{\alpha}$. Thus since $\mathscr{H}$ has cardinality $c$ and $c f(c)>\omega_{1}$ there is an $f \in \mathscr{S}$ and an $\alpha \in \omega_{1}$ such that $D_{f}^{\alpha}$ includes $c$ members of $\mathscr{H}$. Let $\mathscr{H}^{\prime}$ be those members of $\mathscr{H}$ which are contained in $D_{f}^{\kappa}$.

THeORem 5.3. Assuming $\mathrm{CH}$, there is a subcollection $\mathscr{\mathscr { C }}$ of $\mathscr{F}$ having cardinality $c$ and such that if $\mathscr{H}^{\prime}$ is any subcollection of $\mathscr{Y}$ with cardinality $c$, then $\cup \mathscr{H}^{\prime}$ has an irrational limit point.

Proof. For each nonnegative integer $n$, let $\left\{x_{0}^{n}, x_{1}^{n}, x_{2}^{n}, \cdots\right\}$ denote the rational numbers in the interval $(n, n+1)$. CH implies there 
is an $\omega_{1}$-scale $\mathscr{S}$. For each $f \in \mathscr{S}$ let $D_{f}=\left\{x_{t}^{n}: t \leqq f(n)\right.$ and $\left.n \in \omega\right\}$. Let $\mathscr{H}=\left\{D_{f}: f \in \mathscr{S}\right\}$. For each subset $\mathscr{C}^{\prime}$ of $\mathscr{H}$ having cardinality $c, \mathscr{S}^{\prime}=\left\{f \in \mathscr{S}: D_{f} \in \mathscr{H}^{\prime}\right\}$ is cofinal in $\mathscr{S}$. Hence $\cup \mathscr{H}^{\prime}$ is dense in $R$.

\section{REFERENCES}

1. H. R. Bennett, On quasi-developable spaces, Gen. Top. and Appl., 1 (1971), 253-262.

2. R. H. Bing, Metrization of topological spaces, Canad. J. Math., 3 (1951), 175-186.

3. C. J. R. Borges, On metrizability of topological spaces, Canad. J. Math., 20 (1968), 795-804.

4. F. Bernstein, Zur Theorie der trigonometrischen Reihen, Leipz. Ber, 60 (1908), 325-338.

5. J. W. Green, Moore-closed spaces, completeness and centered bases, Gen. Top. and Appl., 4 (1974), 297-313.

6. Moore-closed and locally Moore-closed spaces, Set Theoretic Topology, Academic Press, 1977, 193-217.

7. F. Hausdorff, Untersuchen über Ordungstypen, Ber. Sachs. Acad. Wiss., 59 (1907), 84-159.

8. S. H. Hechler, On the existence of certain cofinal subsets of ${ }^{\omega} \omega$, Proc. Sympos. Pure Math., vol. 13, part 2, Amer. Math. Soc., Providence, R. I., 1974, 155-173.

9. ㄴ On a ubiquitous cardinal, Proc. Amer. Math. Soc., 52 (1975), 348-352.

10. R. E. Hodel, Moore spaces and w4-spaces, Pacific J. Math., 38 (1971), 641-651.

11. - Spaces defined by sequences of open covers which guarantee that certain sequences have cluster points, Duke Math. J., 39 (1972), 253-263.

12. T. J. Jech, Lectures in Set Theory with Particular Emphasis on the Method of Forcing, Springer-Verlag Lect. Notes in Math., Vol. 217, 1971.

13. R. L. Moore, Foundations of Point Set Theory, Amer. Math. Soc. Colloq. Pub., Vol. 13 rev. ed., 1962.

14. T. R. Pettis, Collections of covers which imply compactness, Ph. D. Thesis, University of Oklahoma.

15. J. H. Roberts, A property related to completeness, Bull. Amer. Math. Soc., 38 (1932), 835-838.

16. D. R. Traylor, Completeness in developable spaces, preprint.

17. H. H. Wicke and J. M. Worrell, Point-countability and compactness, Proc. Amer. Math. Soc., 55, No. 2, (1976), 427-431.

18. S. Willard, General Topology, Addison-Wesley, Reading, Mass., 1970.

Received September 3, 1980 and in revised form December 15, 1980.

TeXas A \& M University

College Station, TX 77843 



\section{PACIFIC JOURNAL OF MATHEMATICS}

\section{EDITORS}

DONALD BABBITT (Managing Editor)

University of California

Los Angeles, CA 90024

Hugo RossI

University of Utah

Salt Lake City, UT 84112

C. C. Moore and Arthur Agus

University of California

Berkeley, CA 94720
J. DugundJI

Department of Mathematics

University of Southern California

Los Angeles, CA 90007

R. FinN and J. MiLgRAM

Stanford University

Stanford, CA 94305

\section{ASSOCIATE EDITORS}
R. ARENS
E. F. BECKENBACH
B. H. NeumanN
F. WOLF
K. YoSHIDA

\section{SUPPORTING INSTITUTIONS}

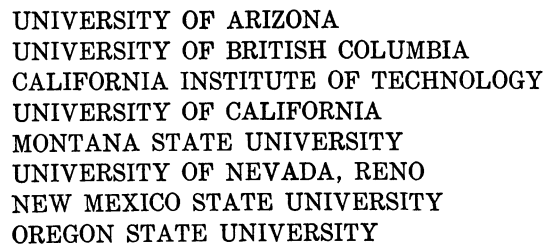

UNIVERSITY OF ARIZONA

UNIVERSITY OF BRITISH COLUMBIA CALIFORNIA INSTITUTE OF TECHNOLOGY UNIVERSITY OF CALIFORNIA MONTANA STATE UNIVERSITY

UNIVERSITY OF NEVADA, RENO NEW MEXICO STATE UNIVERSITY OREGON STATE UNIVERSITY

\author{
UNIVERSITY OF OREGON \\ UNIVERSITY OF SOUTHERN CALIFORNIA \\ STANFORD UNIVERSITY \\ UNIVERSITY OF AAWAII \\ UNIVERSITY OF TOKYO \\ UNIVERSITY OF UTAH \\ WASHINGTON STATE UNIVERSITY \\ UNIVERSITY OF WASHINGTON
}

The Supporting Institutions listed above contribute to the cost of publication of this Journal, but they are not owners or publishers and have no responsibility for its content or policies,

Mathematical parers intended for publication in the Pacific Journal of Mathematics should be in typed form or offset-reproduced, (not dittoed), double spaced with large margins. Please do not use built up fractions in the text of the manuscript. However, you may use them in the displayed equations. Underline Greek letters in red, German in green, and script in blue. The first paragraph or two must be capable of being used separately as a synopsis of the entire paper. Please propose a heading for the odd unmbered pages of less than 35 characters. Manuscripts, in triplicate, may be sent to any one of the editors. Please classify according to the scheme of Math. Reviews, Index to Vol. 39. Supply name and address of author to whom proofs should be sent. All other communications should be addressed to the managing editor, or Elaine Barth, University of California, Los Angeles, California, 90024 .

50 reprints to each author are provided free for each article, only if page charges have been substantially paid. Additional copies may be obtained at cost in multiples of 50 .

The Pacific Journal of Mathematics is issued monthly as of January 1966, Regular subscription rate: $\$ 114.00$ a year (6 Vol., 12 issues). Special rate: $\$ 57.00$ a year to individual members of supporting institution.

Subscriptions, orders for numbers issued in the last three calendar years, and changes of address shoud be sent to Pacific Journal of Mathematics, P.O. Box 969, Carmel Valley, CA 93924, U.S.A. Old back numbers obtainable from Kraus Periodicals Co., Route 100, Millwood, NY 10546.

\section{PUBLISHED BY PACIFIC JOURNAL OF MATHEMATICS, A NON-PROFIT CORPORATION}

Printed at Kokusai Bunken Insatsusha (International Academic Printing Co., Ltd.). 8-8, 3-chome, Takadanobaba, Shinjuku-ku, Tokyo 160, Japan.

Copyright (C) 1982 by Pacific Journal of Mathematics Manufactured and first issued in Japan 


\section{Pacific Journal of Mathematics}

Vol. 100, No. $2 \quad$ October, 1982

Kenneth F. Andersen, On the transformation of Fourier coefficients of

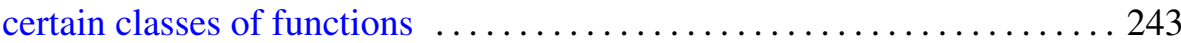

Steven Albert Bleiler, Realizing concordant polynomials with prime knots

Reinhard Bürger, Functions of translation type and solid Banach spaces of functions

Ulrich Daepp, The saturation of $k$-analytic rings and topological equivalence of associated analytic set germs .................. 271

Persi W. Diaconis and David Amiel Freedman, On the maximum difference between the empirical and expected histograms for sums . . . 287

David Amiel Freedman, On the maximum of scaled multinomial

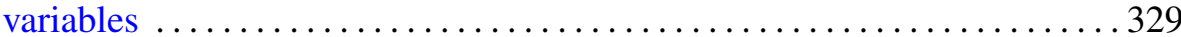

Persi W. Diaconis and David Amiel Freedman, On the difference between the empirical histogram and the normal curve, for sums. II ......... 359

Persi W. Diaconis and David Amiel Freedman, On the mode of an

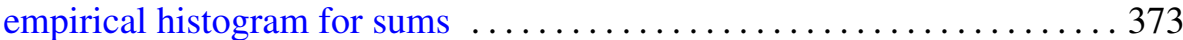

Jutta Hausen, Supplemented modules over Dedekind domains 387

Elyahu Katz, A moduli representation for the classification of twisted tensor

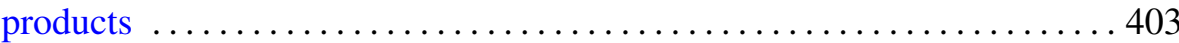

H. C. Madhekar and N. K. Thakare, Biorthogonal polynomials suggested by the Jacobi polynomials

Ted R. Pettis, Collections of covers of metric spaces 425

Ryōtarō Satō, Maximal functions for a semiflow in an infinite measure space

Michael Jay Stob, Invariance of properties under automorphisms of the lattice of recursively enumerable sets 